modèles $\cdot \cdots \cdot \cdot \cdot$ ! !

ling uistiques

\section{Modèles linguistiques}

$53 \mid 2006$

La préposition en français (I). Philologie et linguistique diachronique (domaine anglais)

\title{
2. A propos de la Sorbonne sous Vichy
}

Jean Martin

\section{OpenEdition}

\section{Journals}

Édition électronique

URL : https://journals.openedition.org/ml/554

DOI : $10.4000 / \mathrm{ml} .554$

ISSN : 2274-0511

Éditeur

Association Modèles linguistiques

\section{Édition imprimée}

Date de publication : 1 janvier 2006

Pagination : 154-157

\section{Référence électronique}

Jean Martin, «2. A propos de la Sorbonne sous Vichy », Modèles linguistiques [En ligne], 53 | 2006, mis en ligne le 01 février 2015, consulté le 01 juillet 2021. URL : http://journals.openedition.org/ml/554 DOI : https://doi.org/10.4000/ml.554

Ce document a été généré automatiquement le 1 juillet 2021

(C) Modèles Linguistiques 


\title{
2. A propos de la Sorbonne sous Vichy
}

\author{
Jean Martin
}

1 Le hasard d'un service de presse a mis sous mes yeux un ouvrage collectif, intitulé : Medieval English Language Scholarship. Autobiographies by Representative Scholars in Our Discipline ${ }^{1}$. Sous le titre: "Brute Beauty and valour and act...", la contribution d'André Crépin, professeur émérite à Paris IV et membre de l'Institut, a retenu mon attention.

2 Retraçant sa carrière à la Sorbonne, l'auteur écrit, à propos de la succession du Professeur Emile Pons (1956) : "His retirement brought about a crisis at the Sorbonne. The obvious successor was Fernand Mossé but the English Faculty had accepted the regime of Pétain and felt reluctant to elect a man fresh from the Gaullist Free Forces. In the Hundred Years War, the Sorbonne had sided with the English and the Burgundians against Joan of Arc; now it preferred to bypass Mossé and to choose an interim member, a colleague from the German Department, Jean Fourquet..." (Son départ en retraite engendra une crise à la Sorbonne. Fernand Mossé était donné comme successeur évident, mais l'Institut d'anglais avait admis le régime de Pétain, et il se montra réticent à élire un candidat frais issu de la France Libre de De Gaulle. De même, pendant la guerre de Cent ans, la Sorbonne avait pris le parti des Anglais et des Bourguignons contre Jeanne d'Arc ; maintenant, elle préférait écarter Fernand Mossé pour élire à titre intérimaire un collègue d'Études germaniques, Jean Fourquet).

Une telle assertion quant à l'adhésion de l'Institut d'anglais au régime de Vichy va à l'encontre des informations que j'avais pu obtenir auprès de vieux professeurs, pour la plupart aujourd'hui disparus, qui avaient vécu cette période, aussi bien que de la lecture de l'ouvrage de Jean-Pierre Mouchon ${ }^{2}$. Ayant enseigné l'Histoire à la Sorbonne pendant quatorze ans (1976-1990), je considère qu'il est de mon devoir de ne pas laisser dénigrer cette institution, ni ternir la mémoire des maîtres qui s'y trouvaient pendant la guerre.

4 A partir de la rentrée de 1940, l'Institut d'anglais ne comptait plus que quatre professeurs (voir ci-dessus la note 3), auxquels une chargée de conférences vint s'adjoindre en janvier 1941. En outre, Mme Marthe Huchon, recrutée comme lectrice, 
vint renforcer cette petite communauté scientifique. Pour celle-ci, la période avait débuté tragiquement, puisque le professeur René Huchon (1872-1940) s'était donné la mort le 25 juin 1940, jour de l'Armistice. Il ne fut pas seul dans ce cas ; que l'on pense au suicide, dans les mêmes temps (14 juin 1940, le jour où Paris fut déclaré ville ouverte), du Dr Thierry de Martel, fils de l'écrivain Gyp et descendant de Mirabeau et, le 20 juin 1940, celui du Dr Georges Basch, fils de Victor Basch, et père de Françoise Basch ${ }^{3}$. Mais ce fait indique, du moins, que R. Huchon ne devait pas saluer avec joie l'invasion de son pays, ni voir une divine surprise dans l'avènement du maréchal Pétain.

5 Entre 1940 et l'été 1944, l'Institut d'anglais vécut des jours pénibles. Les anglicistes étaient tout naturellement suspectés de sympathies pour les Anglo-Saxons et, comme tels, en proie à la surveillance des autorités allemandes et des agents du régime de Vichy. Cette vigilance n'était pas infondée.

6 Le professeur Floris Delattre (1880-1950) avait épousé une Juive, Henriette Laure Eisenschitz, nièce de Bergson, et il fut un familier ${ }^{4}$ de ce dernier. Son épouse porta l'étoile jaune. Leur fils, Jacques, étudiant en médecine, put, en 1943, gagner Alger et rejoindre la France combattante grâce à une filière d'évasion, organisée par un haut fonctionnaire des Finances qui n'était autre que le père de Marguerite-Marie Dubois, chargée de cours à la Sorbonne. Le professeur Charles Cestre (1871-1958) hébergea un temps le jeune résistant Roland Dumas, futur ministre, dont la carrière est bien connue. Longtemps plus tard, le 18 décembre 1985, Roland Dumas devait organiser à la Sorbonne une cérémonie à la mémoire de son protecteur. Un frère de Charles Cestre, l'ingénieur en chef des Ponts et Chaussées Louis Cestre, fut déporté à Buchenwald en 1943. Le gendre de Charles Cestre, Jean Rist, qui appartenait à la Résistance, tomba sous les balles ennemies au mois d'août 1944, dans la Loire, lors d'un engagement entre les F.F.I. et les troupes allemandes qui refluaient vers le front de Normandie. Peu auparavant, au printemps 1944, le professeur Edouard Guyot (1884-1948), résistant notoire, avait été sauvagement agressé par les miliciens cantonnés au lycée Saint-Louis, et il devait mourir des suites de ses blessures quatre ans plus tard.

7 Telle fut la prétendue brochette de vichystes et de collaborateurs qui, aux dires de l'éminent angliciste, peuplait l'Institut d'anglais de la Sorbonne pendant ces années noires.

8 Un doute pourrait à la rigueur subsister en ce qui concerne le professeur Louis Cazamian (1877-1965). Ce dernier était un pacifiste invétéré qui refusait obstinément de prendre parti en faveur de l'un ou l'autre des deux camps en présence. Admirateur de l'ordre et de la tenue des armées allemandes (comme Gide le fut à Tunis à la même période), il ne se gêna pas pour réprouver les bombardements alliés. Cette attitude lui valut une sanction (suspension le 21 septembre 1944 et retraite d'office le 24 janvier 1945) dont il fut ensuite relevé à la grande satisfaction de certains collègues d'Oxford qui, le tenant en estime, avaient souligné qu'aucun fait de collaboration ne pouvait être retenu à son encontre.

9 Nous n'insisterons pas sur le rapprochement que fait M. Crépin entre la Sorbonne de l'époque de Jeanne d'Arc et celle des années 1940-1944. L'Histoire ne se répète pas, et une telle comparaison n'est qu'une parfaite coquecigrue (qui indiquerait cependant que, dès ces temps lointains, la Sorbonne avait des sympathies pour la cause de l'Angleterre!). Le thème de la collusion avec l'ennemi est moins nouveau qu'il n'y paraît : vers 1910, L'Action Française de Maurras reprochait déjà à Charles Andler de propager la pensée allemande dans ses cours. M. André Crépin pourrait se reporter 
avec profit à Jean Guéhenno qui rendit naguère au patriotisme des professeurs de la Sorbonne pendant la dernière guerre, un hommage mérité.

On pourrait encore remarquer qu'en 1956, au moment de la succession mentionnée dans l'article, deux des électeurs étaient morts et les deux autres en retraite, et qu'ils n'étaient donc plus là pour faire échec au recrutement de Fernand Mossé. Vétéran de 14-18, ce dernier avait fait une brillante campagne de 39-40 (il prit part à la fameuse bataille de l'eau lourde), mais il n'a pas appartenu aux Forces Françaises Libres, n'ayant rejoint De Gaulle ni à Londres, ni à Alger. (On désigne sous le nom de Forces Françaises Libres les troupes mises sur pied par De Gaulle outre-mer afin de poursuivre la lutte aux côtés des Alliés; ces troupes s'illustrèrent en Libye, en Tunisie, en Italie et en d'autres lieux). Mossé ne peut donc être qualifié de fresh from the Gaullist Free Forces ». (Nul ne pourrait d'ailleurs être qualifié de "fresh onze ans après la fin des hostilités !). Enfin, dois-je rappeler que Mossé mourut le 10 juillet 1956, quelques jours avant le départ en retraite d'Émile Pons, le 19 juillet 1956 ?

D'autres professeurs avaient pris la relève, qui n'étaient pas plus pétainistes que leurs prédécesseurs, parmi lesquels Raymond Las Vergnas, ancien prisonnier de guerre et adversaire déterminé du régime de Vichy. De plus, nul n'ignore que Fernand Mossé était, depuis 1949, professeur au Collège de France (l'article le mentionne) et, eût-il été vivant, il n'aurait plus eu aucune raison de briguer une chaire à la Sorbonne ${ }^{5}$.

La science historique est une maitresse exigeante, avec laquelle il est déconseillé de badiner.

\section{NOTES}

1. Voir ci-dessus les références complètes de cet ouvrage dans la note 1 de l'article d'A. Joly.

2. J.-P. Mouchon, Esquisse de l'enseignement de l'anglais et des études anglaises en France au $\mathrm{xx}^{\mathrm{e}}$ siècle, Marseille : Terra Beata, 1995.

3. Cf. Françoise Basch, Victor Basch, de l'Affaire Dreyfus au crime de la Milice, Paris, Plon, 1994. Françoise Basch a enseigné la civilisation anglo-américaine à l'Université Paris VII-Denis Diderot.

4. Le premier volume des Etudes bergsoniennes (Bergson et Proust, Paris, Albin Michel, 1948) est signé par Floris Delattre.

5. En fait, M. Crépin situe en 1956 (succession d'Emile Pons) des faits qui remontent à 1940 (succession de René Huchon)... 


\section{AUTEUR}

JEAN MARTIN

Professeur émérite à l'Université de Lille III - Ancien maître de conférences d'Histoire à la Sorbonne (Paris IV) 